\title{
Revealing the retrodonation effect by the chemical bond overlap model in organometallic systems
}

Carlos V. Santos Jr. ${ }^{1}(\mathrm{IC})^{*}$, Albano N. Carneiro Neto ${ }^{2}$ (PG), Renaldo T. Moura Jr. ${ }^{1}$ (PQ). carlos1995junior@gmail.com

${ }^{1}$ Department of Chemistry and Physics, Center of Agrarian Sciences, Federal University of Paraíba, Campus II - University City, 58397-000 Areia, PB, Brazil

${ }^{2}$ Department of Fundamental Chemistry, Federal University of Pernambuco, University City, 50670-901 Recife, PE, Brazil

Keywords: Organometallic, chemical bond, overlap.

\section{Introduction}

Organometallic compounds are widely used both in research and industrial chemical reactions, as well as in the role of catalysts to increase the rates of different type of reactions (e.g., as in uses of homogeneous catalysis), where target molecules include polymers, pharmaceuticals, and many other types of practical products. One of the main electronic effect at the organometallic compounds that contribute to the increase of the reactivity of the ligands is the retrodonation effect, that activates, for instance, the $\mathrm{CO}$ ligand coordinated to a central metal or ion. The stretching frequency $(v)$ of $\mathrm{CO}$ is used to infer about the retrodonation character in organometallic compounds, where the higher the frequency the smaller is the retrodonation. This work aims to study the $\mathrm{CO}$ and $\mathrm{C}=\mathrm{C}$ chemical bonds in organometallic compounds by the application of the chemical bond overlap model (OP) [1]. This concept has been introduced and led to relevant conclusions on the interpretation of covalence in chemical bonds. It was recently developed in our group a methodology for calculating the chemical bond overlap properties that uses localized molecular orbitals, which makes possible to calculate the chemical bond overlap properties (i.e. overlap polarizabilities, $\alpha_{\mathrm{OP}}$ ).

\section{Methodology}

To makes it possible the analysis of overlap properties of chemical bonds in organometallic systems, it was used the methodology developed in our group. The Bond

$$
\rho_{\mathrm{OP}}(r)=\sum_{i \in A}^{m} \sum_{j \in B}^{m} c_{i} c_{j} \varphi_{i} \varphi_{j}
$$

Overlap Polarizability Package (BOPP) is a program that was developed to calculate the overlap properties using localized molecular orbitals. The Eq. 1 and 2 are implemented in BOPP and allow the calculation of the overlap and density $(p)$ and polarizability $\left(\alpha_{\mathrm{op}}\right)$.

$$
\alpha_{\mathrm{OP}}^{\kappa \lambda}=-\frac{2}{F_{\kappa}}\left(\sum_{\frac{i \in A}{i \in B}}^{m} \sum_{\frac{j \in B}{j \in A}}^{n} c_{i}^{\prime} c_{j}^{\prime}\left\langle\varphi_{i}|\lambda| \varphi_{j}\right\rangle-\sum_{\frac{i \in A}{i \in B}}^{m} \sum_{\substack{j \in B \\ j \in A}}^{n} c_{i}^{0} c_{j}^{0}\left\langle\varphi_{i}|\lambda| \varphi_{j}\right\rangle\right)^{\prime} \quad \kappa, \lambda=x, y, z
$$

It is possible to generate overlap density maps, creating files in Gaussian cube format. This files can be read in visualization programs (as Chemcraft or VESTA), allowing the creation of nice images for a best qualitative understanding of the properties calculated.

The studied systems were $\left[\mathrm{M}(\mathrm{CO})_{6}\right]$, where $\mathrm{M}=\mathrm{Ti}, \mathrm{V}, \mathrm{Cr}, \mathrm{Mn}, \mathrm{Fe}, \mathrm{Co}, \mathrm{Ni}, \mathrm{Cu}$. The total charge of each system was adjusted to keep the number of electrons equal to 108. The geometry optimization was performed with the GAMESS program, using B3LYP/aug-cc-pVQZ and wB97X-D/aug-cc-pVQZ methods. The orbital localization 
was made using the Pipek-Mezey and Ruedenberg methods. The overlap properties calculations were made in our BOPP software.

\section{Results and Discussion}

The obtained results are schematized in Fig. 1, where the overlap polarizabilities and overlap charges for the different systems are depicted. Fig. 1a shows the overlap polarizability for the metal-ligand bonds, the $\sigma$-type and two $\pi$-type $\mathrm{C}-\mathrm{O}$ bonds in the ligand $\mathrm{CO}$, while their overlap charge density are depicted in Fig. 1b.

Figure 1. Obtained overlap properties for the studied systems.

a)

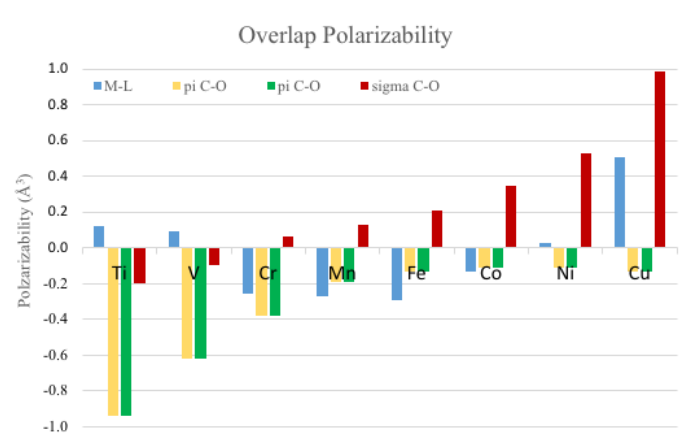

b)

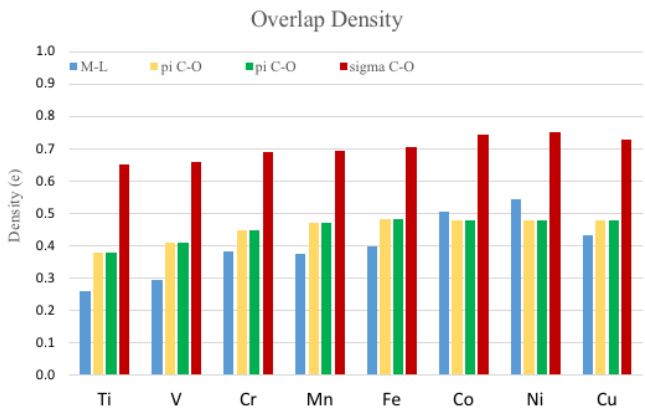

First is emphasized that the number of electrons in all of the systems is the same, while the metal atomic number $\mathrm{Z}$ is increasing. As a consequence, from $\mathrm{Ti}$ to $\mathrm{Cu}$, the nuclear effective charge $\left(Z_{\text {eff }}\right)$ is increasing and it is expected a decrease at the retrodonation effect, as it can be seen in the experimental stretching frequency of $\mathrm{CO}$, that increases in function of the metal $\left(v_{\mathrm{CO}}(\mathrm{M})\right.$, where $v_{\mathrm{CO}}(\mathrm{Ti})=1748 \mathrm{~cm}^{-1} ; v_{\mathrm{CO}}(\mathrm{V})=1859$ $\left.\mathrm{cm}^{-1} ; v_{\mathrm{CO}}(\mathrm{Cr})=2000 \mathrm{~cm}^{-1} ; v_{\mathrm{CO}}(\mathrm{Mn})=2100 \mathrm{~cm}^{-1}\right)$. The overlap properties is sensitive to this trend as follow: as the metal $\mathrm{Z}_{\text {eff }}$ increases, the overlap density at the $\mathrm{M}-\mathrm{L}$ bond is shifted to M (As can be seen in Fig. 2), while its polarizability decrease from Ti to Fe. The consequence of this shift is that the $\mathrm{C}-\mathrm{O}$ bonds become less repelled by the carbon lone pair (the $\sigma$-donator), possessing bigger overlap polarizabilities and charges in the direction $\mathrm{Ti} \rightarrow \mathrm{Cu}$. These and other issues will be addressed during the event.

Figure 2. Populational maps generating in the BOPP for systems studied.
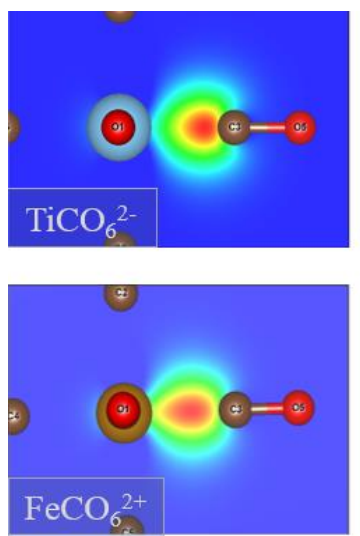
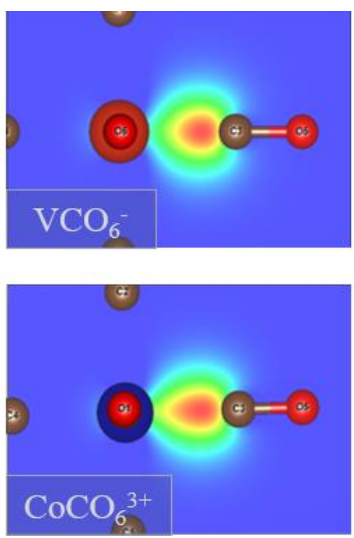
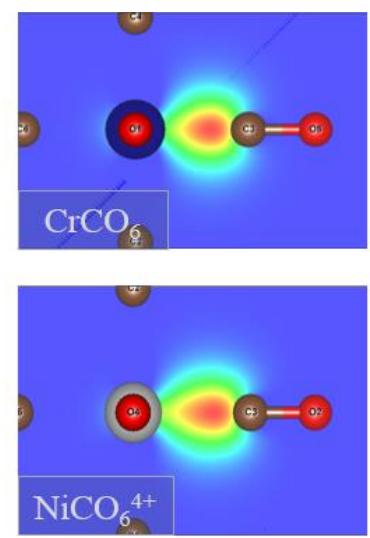
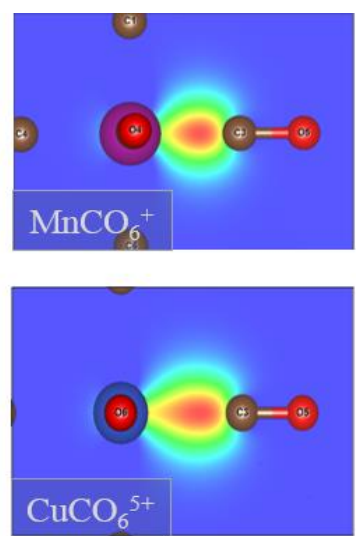

\section{Acknowledgments}

We deeply thank the CNPq, and LQTC-UFPE for the support.

\section{References}

[1] O.L. Malta, H.J. Batista, L.D. Carlos, Chem. Phys. 21282 (2002). 